\title{
CONTIGUOUS SPHERE CLUSTERS. I. ISOMORPHIC CHAINS
}

\author{
FRED SUPNICK
}

One of the remarkable phenomena (still unexplained) concomitant with the transmission of the "code of heredity" to succeeding generations (in sexually reproducing organisms) is "pairing" of the chromosomes at zygotene: That is, during the zygotene stage of meiosis (the process whereby the sex cells are formed) homologous chromosomes become "contiguous" at homologous sites (the chromomers) throughout their length.

In this paper we consider an analogous geometric situation using "pairing at zygotene" as our prototype. Our "geometric chromosome" will be a "chain" of (not necessarily equal) spheres. Now, the fact that two "isomorphic" sphere chains (i.e. "twins") can achieve (simultaneous) contiguity between corresponding spheres, has implications for the structure of the chains. Thus, e.g., what about the relative sizes of the constituent spheres? It is the purpose of the present study to derive some of these structural implications.

1. Definitions and statement of the theorem. Let $s_{i}$ and $c_{i}$ denote the radius and center of the 3 -sphere $S_{i}$ respectively. Let $l\left(c_{i}, c_{j}\right)$ denote the line passing through the points $c_{i}$ and $c_{j}$.

By a twin-chain $\left[S_{1}, \cdots, S_{n} ; S_{1}^{\prime}, \cdots, S_{n}{ }^{\prime}\right]$ of spheres we shall mean a configuration of (Euclidean) 3-spheres

$$
S_{1}, S_{2}, \cdots, S_{n}, S_{1}^{\prime}, S_{2}^{\prime}, \cdots, S_{n}^{\prime}
$$

such that (i) no two spheres of (1.1) have interior points in common, (ii) $s_{i}=s_{i}^{\prime}(i=1, \cdots, n)\left(s_{i}^{\prime}\right.$ is the radius of $\left.S_{i}^{\prime}\right)$, and (iii) $S_{i}$ is tangent to $S_{i+1}(i=1, \cdots, n-1), S_{i}^{\prime}$ is tangent to $S_{i+1}^{\prime}$ $(i=1, \cdots, n-1)$, and $S_{i}$ is tangent to $S_{i}^{\prime}(i=1, \cdots, n)$.

Twin-chains $\left[S_{1}, \cdots, S_{n} ; S_{1}^{\prime}, \cdots, S_{n}^{\prime}\right]$ and $\left[T_{1}, \cdots, T_{n}\right.$; $\left.T_{1}^{\prime}, \cdots, T_{n}^{\prime}\right]$ will be referred to as equivalent if $s_{i}=t_{i}(i=1, \cdots, n)$ $\left(t_{i}\right.$ is the radius of $\left.T_{i}\right)$.

If spheres (1.1) are "moved" (possibly with varying mutual relative positions) so that at each instant $t$ of a time interval $0 \leqq t \leqq 1$, properties (i), (ii), (iii) (in the definition of twin-chain) hold, then we shall say that the twin-chain $K(0):\left[S_{1}, \cdots, S_{n} ; S_{1}^{\prime}, \cdots, S_{n}^{\prime}\right]_{0}$ at $t=0$ has been deformed into the twin-chain $K(1):\left[S_{1}, \cdots, S_{n}\right.$; $\left.S_{1}^{\prime}, \cdots, S_{n}^{\prime}\right]_{1}$ at $t=1$; we shall refer to $K(1)$ as a deform of $K(0)$.

Received by the editors August 29, 1960. 
$K(0)$ and $K(1)$ are, of course, equivalent. The set of all possible deforms of $K(0)$ will be referred to as a deformation class. Now, let $S$ be the set of all twin-chains which are equivalent to a given twinchain $K$. Then $S$ decomposes into deformation classes such that if $X, Y \in S$, then $X$ and $Y$ belong to the same deformation class if and only if $X$ is a deform of $Y$.

We shall refer to " $n$ " (the highest subscript appearing in the bracket symbol for a twin-chain) as the length of the chain.

Now, all equivalent twin-chains of length two belong to the same deformation class. However, not all equivalent twin-chains of length three necessarily belong to the same deformation class. Consider the following example: On a Cartesian coordinate system in $E_{3}$ let $S_{1}, S_{2}$, $S_{1}^{\prime}, S_{2}^{\prime}$ be defined as follows:

$S_{1}:(x+1)^{2}+y^{2}+(z-1)^{2}=1, \quad S_{1}^{\prime}:(x-1)^{2}+y^{2}+(z-1)^{2}=1$,

$S_{2}:(x+1)^{2}+y^{2}+(z+1)^{2}=1, \quad S_{2}^{\prime}:(x-1)^{2}+y^{2}+(z+1)^{2}=1$.

Let

$$
T_{3}: x^{2}+(y-R)^{2}+z^{2}=R^{2}, \quad T_{3}^{\prime}: x^{2}+(y+R)^{2}+z^{2}=R^{2}
$$

be spheres each tangent to $S_{1}, S_{2}, S_{1}^{\prime}, S_{2}^{\prime}$, and

$$
\begin{aligned}
& U_{3}: x^{2}+(y-R)^{2}+(z-\alpha)^{2}=R^{2}, \\
& U_{3}^{\prime}: x^{2}+(y+R)^{2}+(z-\alpha)^{2}=R^{2}
\end{aligned}
$$

$(\alpha<0)$ be spheres each tangent to $S_{2}$ and $S_{2}^{\prime}$. Since

$$
\left[S_{1}, S_{2}, T_{3} ; S_{1}^{\prime}, S_{2}^{\prime}, T_{3}^{\prime}\right]
$$

permits no deformation (except those deforms which belong to the same congruence class) and

$$
\left[S_{1}, S_{2}, U_{3} ; S_{1}^{\prime}, S_{2}^{\prime}, U_{3}^{\prime}\right]
$$

does, (1.2) and (1.3) belong to distinct deformation classes.

A twin-chain $\left[S_{1}, S_{2}, S_{3} ; S_{1}^{\prime}, S_{2}^{\prime}, S_{3}^{\prime}\right]$ such that

(i) the points $P_{i}$ of contact between $S_{i}$ and $S_{i}^{\prime}(i=1,2,3)$ are collinear on a line $L$,

(ii) $l\left(c_{i}, c_{i}^{\prime}\right)\left(c_{i}^{\prime}\right.$ the center of $\left.S_{i}^{\prime}\right)$ is perpendicular to $L(i=1,2,3)$,

(iii) $l\left(c_{1}, c_{1}^{\prime}\right)$ is perpendicular to $l\left(c_{3}, c_{3}^{\prime}\right)$,

will be called normal. We shall refer to a normal twin-chain $\left[S_{1}, S_{2}, S_{3} ; S_{1}^{\prime}, S_{2}^{\prime}, S_{3}^{\prime}\right]$ as being of types $(1,2,3)$ or $(1,3,2)$ accordingly as $P_{2}$ falls between $P_{1}$ and $P_{3}$, or $P_{3}$ falls between $P_{1}$ and $P_{2}$ on $L$.

We prove the following theorem: 
Theorem. (i) If $\left[S_{1}, S_{2}, S_{3} ; S_{1}^{\prime}, S_{2}^{\prime}, S_{3}^{\prime}\right]$ is normal of type $(1,2,3)$, then

$$
s_{2} \geqq \frac{s_{1} s_{3}\left(s_{1}+s_{3}-\left\{2 s_{1} s_{3}\right\}^{1 / 2}\right)}{2\left(s_{1}^{2}+s_{3}^{2}\right)} .
$$

(ii) If $s_{1}, s_{2}, s_{3}$ are positive numbers such that (1.4) holds, then it is possible to construct normal twin-chains $\left[S_{1}, S_{2}, S_{3} ; S_{1}^{\prime}, S_{2}^{\prime}, S_{3}^{\prime}\right]$ of type $(1,2,3)$, where $s_{1}, s_{2}, s_{3}$ are the radii of $S_{1}, S_{2}, S_{3}$ respectively.

Corollary 1. If $\left[S_{1}, S_{2}, S_{3} ; S_{1}^{\prime}, S_{2}^{\prime}, S_{3}^{\prime}\right]$ is normal of type $(1,2,3)$, then $s_{2} \geqq\left\{\left(2-2^{1 / 2}\right) / 4\right\} \cdot \operatorname{Min}\left(s_{1}, s_{3}\right)$.

From the manner in which a twin-chain can be deformed, it may be possible to obtain certain estimates of the relative sizes of its constituent parts:

Corollary 2. If $\left[S_{1}, \cdots, S_{n} ; S_{1}^{\prime}, \cdots, S_{n}^{\prime}\right]$ can be deformed so that for some $i(1<i<n)\left[S_{i-1}, S_{i}, S_{i+1}, S_{i-1}^{\prime}, S_{i}^{\prime}, S_{i+1}^{\prime}\right]$ becomes normal, then (1.4) holds with $i-1, i, i+1$ replacing the subscripts $1,2,3$ respectively.

2. Proof of (i). Let $A, B, C, A^{\prime}, B^{\prime}, C^{\prime}, a, b, c$ denote $S_{1}, S_{2}, S_{3}$, $S_{1}^{\prime}, S_{2}^{\prime}, S_{3}^{\prime}, s_{1}, s_{2}, s_{3}$ respectively. $a, b, c$ are positive by hypothesis; there is no loss in generality if we assume $a \geqq c$ (since in the contrary case we can reletter the spheres).

Let a three-dimensional cartesian coordinate system be superimposed on $\left[A, B, C ; A^{\prime}, B^{\prime}, C^{\prime}\right]$ (which is normal, of type $(1,2,3)$ ) so that the centers of $A, B, C, A^{\prime}, B^{\prime}, C^{\prime}$ are

$$
(-a, 0,0),(-l,-m, n),(0,-c, u)(a, 0,0),(l, m, n),(0, c, u)
$$

respectively, with

$$
u>n>0 .
$$

This uniquely determines the orientation (i.e. the right or left handedness) of the system.

Now, $l^{2}+m^{2}=b^{2}$. Since $A^{\prime}$ and $B^{\prime}$ are tangent, and $B^{\prime}$ and $C^{\prime}$ are tangent, we have

$$
(l-a)^{2}+m^{2}+n^{2}=(a+b)^{2}
$$

and

$$
l^{2}+(m-c)^{2}+(u-n)^{2}=(b+c)^{2}
$$

and thus 


$$
n=\{2 a(b+l)\}^{1 / 2}
$$

and

$$
u=n+\{2 c(b+m)\}^{1 / 2} .
$$

Since $A^{\prime}$ and $C^{\prime}$ have no interior points in common, we have $a^{2}+c^{2}$ $+u^{2} \geqq(a+c)^{2}$, which implies

$$
u \geqq(2 a c)^{1 / 2} .
$$

Combining (2.3), (2.4) and (2.5), we obtain

$$
\left(\frac{b+m}{a}\right)^{1 / 2}+\left(\frac{b+\eta}{c}\right)^{1 / 2} \geqq 1 .
$$

Also, since $A$ and $B^{\prime}$ have no interior points in common, and $C$ and $B^{\prime}$ have no interior points in common, we have

$$
(l+a)^{2}+m^{2}+n^{2} \geqq(a+b)^{2}
$$

and

$$
l^{2}+(m+c)^{2}+(n-u)^{2} \geqq(b+c)^{2}
$$

which imply $l \geqq 0$ and $m \geqq 0$, respectively.

Thus, the normalcy of $\left[A, B, C ; A^{\prime}, B^{\prime}, C^{\prime}\right]$ implies

$$
\begin{gathered}
\left(\frac{b+m}{a}\right)^{1 / 2}+\left(\frac{b+l}{c}\right)^{1 / 2} \geqq 1, \\
m^{2}+l^{2}=b^{2}, \\
l \geqq 0, \quad m \geqq 0 .
\end{gathered}
$$

We shall prove (i) of the theorem, by showing that (2.6) implies

$$
b \geqq \frac{a c\left(a+c-\{2 a c\}^{1 / 2}\right)}{2\left(a^{2}+c^{2}\right)} .
$$

If in (2.6) we let

$$
x=\frac{b+m}{a}, \quad y=\frac{b+l}{c},
$$

we obtain

$$
\begin{gathered}
x^{1 / 2}+y^{1 / 2} \geqq 1, \\
\frac{(x-b / a)^{2}}{(b / a)^{2}}+\frac{(y-b / c)^{2}}{(b / c)^{2}}=1, \\
x \geqq b / a, \quad y \geqq b / c .
\end{gathered}
$$


We now note that if $x_{0}, y_{0}, b_{0}$ satisfies (2.9), then

$$
m_{0} \equiv a x_{0}-b_{0}, \quad l_{0} \equiv c y_{0}-b_{0}, \quad b_{0}
$$

satisfies (2.6). Also, if $m_{0}, l_{0}, b_{0}$ satisfies (2.6), then

$$
x_{0} \equiv \frac{b_{0}+m_{0}}{a}, \quad y_{0} \equiv \frac{b_{0}+l_{0}}{c}, \quad b_{0}
$$

satisfies (2.9). Thus if we find a triplet $(x, y, b)$ satisfying (2.9) with minimal positive $b$, we obtain through $(2.10)$ a triplet $(m, l, b)$ satisfying (2.6) with minimal positive $b$.

On a two-dimensional cartesian coordinate system, let $R$ denote the set of all points for which $x^{1 / 2}+y^{1 / 2} \geqq 1$.

Let $E_{b}$ denote the second expression of $(2.9) ; E_{b}$ is a single parameter family of ellipses, with $b$ the parameter. Each member of $E_{b}$ has its center at $(b / a, b / c)$ with axes of symmetry parallel to the $x$-and $y$-axes, and is tangent to the $x$ - and $y$-axes at the points $(b / a, 0)$ and $(0, b / c)$ respectively. Since $a \geqq c$, the line $y=a x / c$ on which the centers of the members of $E_{b}$ lie, has slope greater or equal to one for any (positive) value of $b$. Each member of $E_{b}$ is "upright", i.e. its major axis is parallel to the $y$-axis. Now, if $(x, y)$ is a point on some member $E_{b_{i}}$ of $E_{b}$ such that $x \geqq b / a, y \geqq b / c$ and $(x, y) \in R$, then $\left(x, y, b_{i}\right)$ will satisfy (2.9).

Now, let $E_{b_{m}}$ be that member of $E_{b}$ which (i) has no interior point in common with $R$, and (ii) is tangent to $x^{1 / 2}+y^{1 / 2}=1$ in a point $P$. It is clear that the abscissa and ordinate of $P$ are greater than or equal to $b_{m} / a$ and $b_{m} / c$ respectively $\left(\left(b_{m} / a, b_{m} / c\right)\right.$ is the center of $\left.E_{b_{m}}\right)$. Since $E_{b_{n}}$, for $b_{n}<b_{m}$, has no point in common with $R$, then $b_{m}$ is the minimum value of $b$ which may be the third member of a triplet $(x, y, b)$ satisfying $(2.9)$.

Now, if $x^{1 / 2}+y^{1 / 2}=1$ and a member of $E_{b}$ are tangent at a point $(x, y)$, then $(x, y)$ must satisfy both equations, and the slopes of each at $(x, y)$ must be equal, i.e. $(x, y)$ must satisfy the system

$$
\begin{gathered}
x^{1 / 2}+y^{1 / 2}=1, \\
\frac{(x-b / a)^{2}}{(b / a)^{2}}+\frac{(y-b / c)^{2}}{(b / c)^{2}}=1, \\
c^{2}(y-b / c) y^{1 / 2}=a^{2}(x-b / a) x^{1 / 2} .
\end{gathered}
$$

Thus, our problem becomes that of finding the smallest value of $b$ for which (2.12) has a solution $(x, y)$.

Now, if we let 


$$
x^{1 / 2}=f, \quad y^{1 / 2}=g,
$$

then (2.12) becomes

$$
\begin{gathered}
f+g=1, \\
\frac{\left(f^{2}-b / a\right)^{2}}{(b / a)^{2}}+\frac{\left(g^{2}-b / c\right)^{2}}{(b / c)^{2}}=1, \\
c^{2}\left(g^{2}-b / c\right) g=a^{2}\left(f^{2}-b / a\right) f .
\end{gathered}
$$

We note that any solution $(f, g, b)$ of (2.14) indicates (through (2.13)) a solution $(x, y, b)$ of $(2.12)$ (since only positive $x, y$ need be considered), and conversely. Therefore a triplet $(f, g, b)$ satisfying (2.14) with minimal $b$ provides a triplet $(x, y, b)$ satisfying (2.12) with minimal $b$.

Now, replacing $f$ by $1-g$ in the second and third equations of (2.14), and simplifying, we obtain

$$
b^{2}+G b+H=0
$$

where

$$
\begin{aligned}
& G=-2(a+c) g^{2}+4 a g-2 a, \\
& H=\left(a^{2}+c^{2}\right) g^{4}-4 a^{2} g^{3}+6 a^{2} g^{2}-4 a^{2} g+a^{2},
\end{aligned}
$$

and

$$
M b+N=0
$$

where

$$
M=-(a+c) g+a, \quad N=\left(a^{2}+c^{2}\right) g^{8}-3 a^{2} g^{2}+3 a^{2} g-a^{2} .
$$

The eliminant between (2.15) and (2.16) is

$$
\left|\begin{array}{ccc}
1 & G & H \\
0 & M & N \\
M & N & 0
\end{array}\right|=0
$$

which becomes

$$
a c g^{2}(g-1)^{2}\left\{2\left(a^{2}+c^{2}\right) g^{2}-4 a^{2} g+2 a^{2}-a c\right\}=0 .
$$

We note that $g=0, g=1$ are the solutions of (2.18) which correspond to the tangency between $x^{1 / 2}+y^{1 / 2}=1$, and members $E_{b_{0}}, E_{b_{1}}$ of $E_{b}$ at the points $(1,0)$ and $(0,1)$ respectively; $b_{0}$ and $b_{1}$ cannot be minimal since $E_{b_{0}}$ and $E_{b_{1}}$ have "internal" points in common with $R$, respectively. Thus, setting the expression in the braces of (2.18) equal to zero, and solving, we obtain 


$$
g=\frac{2 a^{2} \pm(a-c)(2 a c)^{1 / 2}}{2\left(a^{2}+c^{2}\right)} .
$$

Solving (2.15) and (2.16) for $b$, we obtain

$$
b=c g^{2}+a(1-g)^{2} \pm(2 a c)^{1 / 2}(1-g) g
$$

and

$$
b=\frac{c^{2} g^{3}+a^{2}(g-1)^{3}}{c g+a(g-1)},
$$

respectively. Thus, for the two values of $g$ given by (2.19) we obtain two values for $b$ given by (2.21), and four more values given by (2.20). Of these six values of $b$ we shall determine the smallest.

Let $b(g)$ denote the function of $g$ which is on the right side of (2.21). Let $g_{+}$denote the expression on the right side of (2.19) taken with the plus sign, and $g_{-}$the same expression taken with the minus sign. We show that

$$
b\left(g_{+}\right) \geqq b\left(g_{-}\right) .
$$

If $a=c$, then (2.22) obviously holds since $g_{+}=g_{-}$. Suppose $a>c$. Writing $V$ for $(a-c)(2 a c)^{1 / 2}$ we have

$$
\begin{aligned}
b\left(g_{+}\right) & =\frac{1}{4\left(a^{2}+c^{2}\right)}\left(\frac{V^{3}+12 a^{2} c^{2} V+8 a^{2} c^{2}\left(a^{2}-c^{2}\right)}{(a+c) V+2 a c(a-c)}\right) \\
& =\frac{a c}{2\left(a^{2}+c^{2}\right)}\left(\frac{(a+c)^{2}+2(a+c)(2 a c)^{1 / 2}+2 a c}{(a+c)+(2 a c)^{1 / 2}}\right) \\
& =\frac{a c\left(a+c+\{2 a c\}^{1 / 2}\right)}{2\left(a^{2}+c^{2}\right)} .
\end{aligned}
$$

In a similar manner we can show that

$$
b\left(g_{-}\right)=\frac{a c\left(a+c-\{2 a c\}^{1 / 2}\right)}{2\left(a^{2}+c^{2}\right)} .
$$

Comparing $b\left(g_{+}\right)$and $b\left(g_{-}\right)$, we see that (2.22) holds.

We now note that since $a, c>0$, and $a \geqq c$, the inequalities

$$
\begin{aligned}
c g_{-}^{2}+a\left(1-g_{-}\right)^{2}+(2 a c)^{1 / 2} & \left(1-g_{-}\right) g_{-} \\
& \geqq c g_{-}^{2}+a\left(1-g_{-}\right)^{2}-(2 a c)^{1 / 2}\left(1-g_{-}\right) g_{-}, \\
c g_{+}^{2}+a\left(1-g_{+}\right)^{2}+(2 a c)^{1 / 2} & \left(1-g_{+}\right) g_{+} \\
& \geqq c g_{-}^{2}+a\left(1-g_{-}\right)^{2}-(2 a c)^{1 / 2}\left(1-g_{-}\right) g_{-},
\end{aligned}
$$


$c g_{+}^{2}+a\left(1-g_{+}\right)^{2}-(2 a c)^{1 / 2}\left(1-g_{+}\right) g_{+}$

hold. Thus

$$
\geqq c g_{-}^{2}+a\left(1-g_{-}\right)^{2}-(2 a c)^{1 / 2}\left(1-g_{-}\right) g_{-}
$$

$$
c g^{2}+a\left(1-g_{-}\right)^{2}-(2 a c)^{1 / 2}\left(1-g_{-}\right) g_{-}
$$

is the least of the four values of $b$ obtained from (2.19) and (2.20). Furthermore (2.24) is positive since

$$
c g_{-}^{2}+a\left(1-g_{-}\right)^{2} \geqq 2(a c)^{1 / 2}\left(1-g_{-}\right) g_{-}>(2 a c)^{1 / 2}\left(1-g_{-}\right) g_{-} .
$$

Finally we note that (2.23) is identical to (2.24). Writing $K$ for $2 a^{2}-(2 a c)^{1 / 2}(a-c)$, and $L$ for $2 c^{2}+(2 a c)^{1 / 2}(a-c)$, we have

$$
\begin{aligned}
c K^{2}+a L^{2} & -(2 a c)^{1 / 2} K L \\
& =2 a^{3} c^{2}+2 a^{2} c^{3}+2 a c^{4}+2 a^{4} c-2 a^{3} c(2 a c)^{1 / 2}-2 a c^{3}(2 a c)^{1 / 2} \\
& =a c\left(a+c-\{2 a c\}^{1 / 2}\right)(K+L) ;
\end{aligned}
$$

dividing the first and last expressions by $(K+L)^{2}$ we see that (2.23) is identical to (2.24). Thus (2.23) is the smallest of the six b's. This completes the proof of part (i) of the theorem.

Proof of Corollary 1. From (1.4) we obtain

$$
\begin{aligned}
s_{2} & \geqq \frac{s_{1}+s_{3}-\left\{2 s_{1} s_{3}\right\}^{1 / 2}}{2\left(\frac{s_{1}}{s_{3}}+\frac{s_{3}}{s_{1}}\right)} \geqq \frac{s_{1}+s_{3}-\left\{2 s_{1} s_{3}\right\}^{1 / 2}}{2\left(\frac{\max \left(s_{1}, s_{3}\right)}{\min \left(s_{1}, s_{3}\right)}+1\right)} \\
& =\left(\frac{s_{1}+s_{3}-\left\{2 s_{1} s_{3}\right\}^{1 / 2}}{2\left(s_{1}+s_{3}\right)}\right) \min \left(s_{1}, s_{3}\right)=\left(1-\frac{\left\{2 s_{1} s_{3}\right\}^{1 / 2}}{s_{1}+s_{3}}\right) \frac{\min \left(s_{1}, s_{3}\right)}{2} .
\end{aligned}
$$

Since $s_{1}+s_{3} \geqq 2\left\{s_{1} s_{3}\right\}^{1 / 2}$, we have $s_{2} \geqq\left\{\left(2-2^{1 / 2}\right) / 4\right\} \cdot \operatorname{Min}\left(s_{1}, s_{3}\right)$.

3. Proof of (ii). Let $a, b, c>0$ with $a \geqq c$ satisfy

$$
b \geqq \frac{a c\left(a+c-\{2 a c\}^{1 / 2}\right)}{2\left(a^{2}+c^{2}\right)} .
$$

We show how to construct a normal twin-chain

$$
\left[A, B, C ; A^{\prime}, B^{\prime}, C^{\prime}\right]
$$

of type $(1,2,3)$ where $a, b, c$ are the radii of $A, B, C$ respectively.

In view of (3.1) we may obtain a triplet $(x, y, b)$ satisfying (2.9). Thus, if $b / c \geqq 1$ (note that $b / c \geqq b / a$ ), any point $(x, y)$ on the "upper right-half" of the ellipse $E_{b}$ (in the present discussion $b$ is fixed) will suffice. If $b / c<1$ then it might be necessary to solve the first two equations of (2.12), which leads to the solution of a quartic, before a 
point $(x, y)$ on the upper right-half of $E_{b}$ and in $R$ may be selected. The triplet $(x, y, b)$ satisfying (2.9) leads to a triplet $(m, l, b)$ satisfying (2.6). Now, let numbers $n$ and $u$ be defined by (2.3) and (2.4), respectively. We note that $u>n>0$; this will imply that the chain (3.2) which we construct below will be of type $(1,2,3)$. (2.5) holds since the first expression of (2.6) implies

$$
\{2 c(b+m)\}^{1 / 2}+\{2 a(b+l)\}^{1 / 2} \geqq(2 a c)^{1 / 2} ;
$$

but the left side of (3.3) is equal to $u$ (by (2.3) and (2.4)).

Now, let the centers of $A, B, C, A^{\prime}, B^{\prime}, C^{\prime}$ be given by (2.1). Making use of (2.6) and/or (2.3), (2.4), (2.5) it may be shown that $A$ and $A^{\prime}, B$ and $B^{\prime}, C$ and $C^{\prime}, A$ and $B, B$ and $C, A^{\prime}$ and $B^{\prime}, B^{\prime}$ and $C^{\prime}$ are respectively tangent, and that no two of these spheres have interior points in common. (E.g., since $n^{2}=2 a(b+l)$, we have $m^{2}+l^{2}-2 a l+a^{2}$ $+n^{2}=a^{2}+2 a b+b^{2}$, which implies that $A$ and $B$ are tangent. Or, since $m \geqq 0$, we have $2 c(b+m) \geqq 2 c(b-m)$; but $(u-n)^{2} \geqq 2 c(b+m)$ $\geqq 2 c(b-m)$; thus $l^{2}+c^{2}+2 c m+m^{2}+(u-n)^{2} \geqq b^{2}+2 b c+c^{2}$ which implies that $C$ and $B^{\prime}$ have no interior points in common; etc.)

We note finally that conditions (i), (ii), (iii) in the definition of a twin-chain (cf. \$1) are obviously satisfied. Thus $\left[A, B, C ; A^{\prime}, B^{\prime}, C^{\prime}\right]$ is a normal twin-chain of type $(1,2,3)$.

Corollary 3. Any twin-chain $\left[S_{1}, S_{2}, S_{3} ; S_{1}^{\prime}, S_{2}^{\prime}, S_{3}^{\prime}\right]$ for which (1.4) holds, has a normal equivalent of type $(1,2,3)$.

City College, New York 